\title{
Controllably coated graphene oxide particles with enhanced compatibility with poly(ethylene-co-propylene) thermoplastic elastomer for excellent photo-mechanical actuation capability.
}

Josef Osicka ${ }^{1}$, Miroslav Mrlik ${ }^{1 *}$, Marketa Ilcikova ${ }^{2,3}$, Igor Krupa ${ }^{4 *}$, Patrik Sobolčiak ${ }^{4}$, Tomáš Plachý ${ }^{1}$, Jaroslav Mosnáček ${ }^{2,5^{*}}$

${ }^{1}$ Centre of Polymer Systems, University Institute, Tomas Bata University in Zlin, Trida T. Bati 5678, 76001 Zlin, Czech Republic

${ }^{2}$ Polymer Institute, Slovak Academy of Sciences, Dubravska cesta 9, 84541 Bratislava 45, Slovakia

${ }^{3}$ Department of Chemistry, Lodz University of Technology, Institute of Polymer and Dye Technology, 90 924, Lodz, Poland

${ }^{4}$ Center for Advanced Materials, Qatar University, P. O. Box 2713, Doha, Qatar

${ }^{5}$ Centre for Advanced Materials Application, Slovak Academy of Sciences, Dúbravska cesta 9, 84511 Bratislava, Slovakia

*corresponding authors: mrlik@utb.cz,igor.krupa@qu.edu.qa, jaroslav.mosnacek@savba.sk

\section{Abstract:}

This paper reports the utilization of the controllable coating via SI-ATRP technique as a promising approach for controlling stimuli-responsive capabilities of graphene oxide (GO) based nanocomposites. Various polymer brushes with controlled molar mass and narrow dispersity were grown from the surface of GO particles. Modification of GO with 
poly(methyl methacrylate) and poly(n-butyl methacrylate) was proved by transmission electron microscopy, thermogravimetric analysis with online FTIR recording and finally by X-ray photoelectron spectroscopy (XPS). Densities of GO-based materials were investigated and conductivity measurements showed the increase values. XPS and Raman shift was used to confirm the GO particles reduction. A compatibility of the filler with propylene-based elastomer was elucidated by melt rheology. The light-induced actuation capability was investigated on composite samples to show, that polymer hybrid particles based on GO have better compatibility with the polymer matrix and thus their proper dispersibility was significantly improved. In addition the plasticizing effect of the short polymer grafts present on the GO filler surface has the crucial impact on the matrix stiffness and thus the ability of composite material to reversibly respond to the external light stimulation.

Keywords: graphene oxide, ATRP, compatibility, Vistamaxx, grafting, stimuli responsive properties

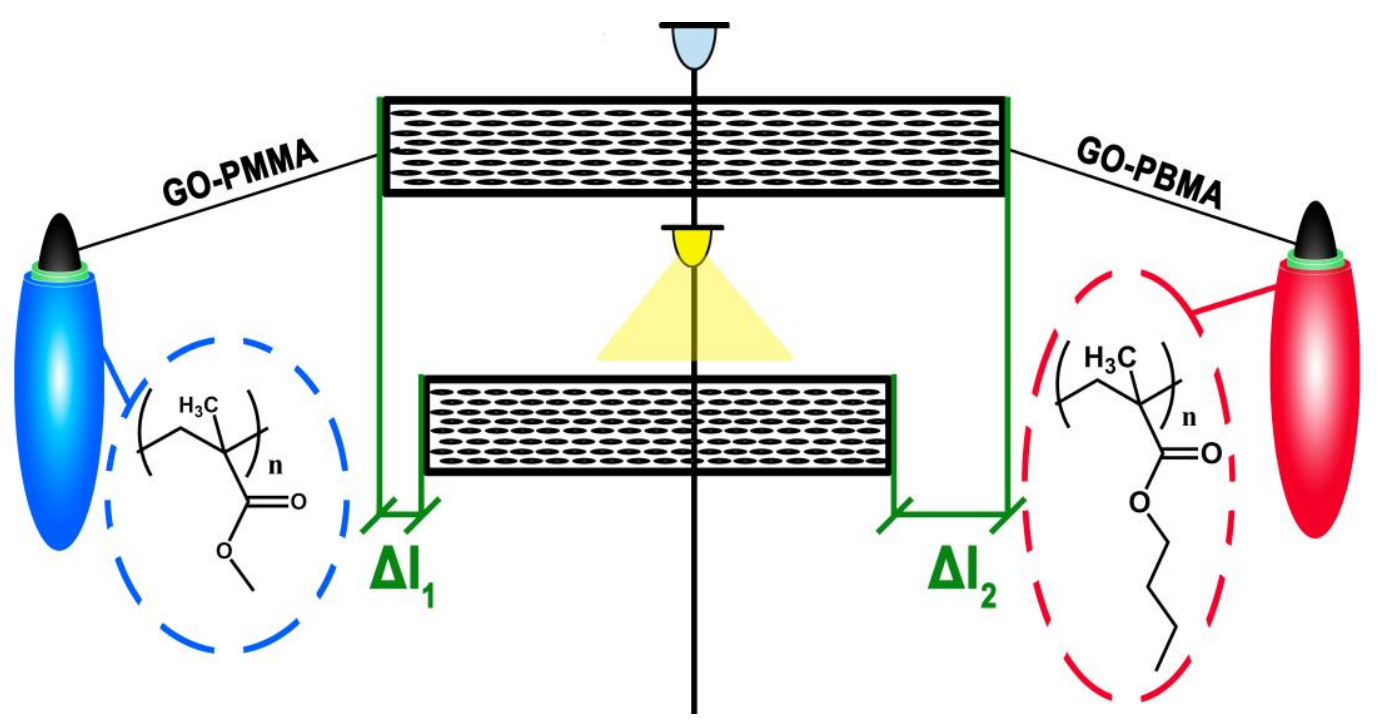




\section{Introduction}

The photomechanical actuators (also known as photo-actuators) are a class of smart materials that can alter their dimensions or undergo mechanical motion when exposed to photons (light emission). Absorption of the energy from photons leads to a contract or a generation of a certain mechanical motion of polymeric chains $[1,2]$, which is called photo-actuation. Photoactuators are kind of promising materials thanks to their wireless actuation. Such behavior can be utilized in many applications, in which a transformation of optical-to-mechanical energy is required or beneficial. Thus, photo-actuating systems are mainly used as various motors [3], as smart curtains [4], systems reproducing biomimetic motions [5], etc. One of the most important class from the application point of view are photo-thermal actuators, which are uniform composite systems consisting of a flexible elastomeric matrix and various functional fillers promoting interactions with photons [2]. Carbon particles such as carbon nanotubes (CNTs) [1,3], graphene oxide [1,6], graphene [7,8] etc., are extensively used as fillers in these systems thanks to their excellent both thermal and mechanical properties. Their utilization increases absorption of the energy from photons [9] and, thus, promotes an overall photomechanical performance of the photo-actuators.

In the most of composite systems, the distribution of particles in the matrix plays an important role in overall performance of the composite together with its photo-actuation performance. It has been shown that the photo-actuating systems based on neat multi-walled CNTs (MWCNTs) exhibited deterioration of the elastic properties of the matrix as confirmed by dynamic mechanic analysis [10]. On the other hand, modification of MWCNTs with polystyrene brushes led to the systems, which preserves flexibility and exhibited sufficient phototactuation of polystyrene- $b$-polyisoprene- $b$-polystyrene nanocomposites [10]. Graphene 
itself, due to its strong aggregation tendency, usually exhibits inhomogeneous distribution within a polymer matrix and, therefore, using of GO is commonly preferred. The presence of functional groups (hydroxyl, epoxy and carbonyl) on the GO surface can be utilized for further modification to target compatibility with wide range of polymer matrices [11]. We have recently introduced [12-14] a surface-initiated atom transfer radical polymerization (SIATRP) as a promising approach for GO surface modification enabling to tune its properties with controlled mechanism of the reaction. SI-ATRP approach can be effectively used for preparation of polymer hybrids enables exact control of polymer architecture including molar mass of the polymers, uniformity of polymer chain length, grafting density, and chain composition $[15,16]$. The surface modification enables the proper filler dispersion, homogenous distribution of the filler within the surrounding polymer, which is crucial for its final performance. Osicka et al. has recently used SI-ATRP for coating of GO particles with poly(glycidyl methacrylate) (PGMA)[17], poly(methyl methacrylate) (PMMA), and poly(nbutyl methacrylate) (PBMA)[18] polymers in form of short brushes. The PMMA and PBMA, compared to neat GO, the increased wettability of the grafted GO hybrids with poly(dimethyl siloxane) matrix was reported. It led to improved particles dispersion and distribution of the filler resulting in increased thermal conductivities of their composites. Thermal conductivity is one of the crucial factor affecting photo-actuation effect [19] and such systems, i.e. containing PMMA and PBMA modified GO, have therefore exhibited more significant photo-actuation effect with the maximum value of the actuation around $35 \mu \mathrm{m}$ under particles loading of 1 wt.\% [18]. Also in the case of PGMA brushes the grafted GO-based systems exhibited increased photo-actuation effect together with higher thermal conductivity. It is worth to note, that better dispersion and homogeneous distribution of the particles within the polymer matrix achieved through modification of GO benefits overall performance of these composites. 
In this study the GO-polymer hybrids were used for the first time for preparation of photomechanical actuators based on a poly(propylene-co-ethylene) matrix possessing thermoplastic elastomer properties. The poly(propylene-co-ethylene) copolymer was chosen as a polymer matrix, due to its easy processing conditions, proper elasticity, long-term stability and enhanced chemical resistance. The GO was grafted with two types of polymer brushes, namely PMMA and PBMA. The effect of presence and type of polymer grafts was investigated using rheological investigations showing better insight into the interactions between GO-based particles and poly(propylene-co-ethylene) copolymer. Finally, photoactuation performance showed that incorporation of the GO-polymer hybrids into the polymeric matrix led to the enhancement in determined value of actuations and thus significantly increased capability of the system.

\section{Experimental part}

\section{Materials}

The sacrificial initiator methyl 2-bromopropionate $98 \%$, initiator for direct covalent bonding 2-Bromopropionyl bromide $97 \%$, ATRP ligand $N, N, N^{\prime}, N^{\prime \prime}, N^{\prime \prime}-$ pentamethyldiethylenetriamine (PMDETA) $98 \%$, copper bromide $(\mathrm{CuBr}) 97 \%$ and anisole $98 \%$, were utilized without further purification. Methyl methacrylate $98 \%$, n-butyl methacrylate $98 \%$ were purified by passing through a basic alumina before the use in order to remove a stabilizer. Triethylamine $\left(\mathrm{Et}_{3} \mathrm{~N}\right) 98 \%$ and tetrahydrofuran (THF) were dried overnight using sodium cubes. All chemical were bought from Sigma Aldrich, USA Graphite in form of fine powder $40 \mu \mathrm{m}$ (Sigma Aldrich, USA) was used as received. Dimethylformamid p.a., acetone p.a. and diethylether p.a. (LachNer, Gemany) were all used as received. Poly(propylene-co-ethylene) copolymer (Vistamaxx ${ }^{\mathrm{TM}}$ 6202, ExxonMobil, USA) 
contains (85 wt.\%) and (15 wt.\%) of isotactic propylene and random ethylene units, respectively. Vistamaxx has following characteristics such as melt flow index (MFI) $7.4 \mathrm{~g} / 10 \mathrm{~min}$ and polymer density $0.861 \mathrm{~g} \cdot \mathrm{cm}^{-3}$ ). GO and GO modified with ATRP initiator (GO-I) were prepared similarly as was already published [18,20, 21] (see ESI).

\section{Grafting of PMMA and PBMA from GO surface}

General procedure for polymer modification of GO surface was as follows: $1.5 \mathrm{~g}$ of GO-I was put into the special type of flask (Schlenk type) with volume of $100 \mathrm{~mL}$ containing a magnetic bar for stirring. Then, the flask was three times filled inert atmosphere and evacuated and followed by addition of anisole $(30 \mathrm{~mL})$ pre-purged with inert gas. Then ethyl $\alpha$-bromoisobutyrate, as a sacrificial initiator with same molarity as $\mathrm{CuBr}$ were added. Further, PMDETA was added in 4-fold molar excess to the sacrificial initiator. The corresponding monomer $(30 \mathrm{~mL})$ in 100 molar excess to sacrificial initiator was bubbled with inert gas and transferred to the flask. To avoid the presence of oxygen the four F-T-P cycles were completed. Finally, $\mathrm{CuBr}$ was put into the flask under inert atmosphere, when the reaction mixture was frozen. The polymerization was performed at $70^{\circ} \mathrm{C}$ for 2 hours. To stop the reaction, Schlenk flask was opened and additional $15 \mathrm{ml}$ of anisole have been added. Compositions of performed reactions were analyzed and summarized in the Table 1 together with the monomer conversion (based on ${ }^{1} \mathrm{H}$ NMR spectra), $M_{\mathrm{n}}$ and dispersity $(\bigoplus$ ) elucidated by GPC. The reaction mixtures including the GO-based powder were filtered using following procedure. The powders were firstly dispersed in $100 \mathrm{ml}$ of DMF, then filtrated using 0.44 $\mu \mathrm{m}$ PTFE filter and followed by washing with acetone $(100 \mathrm{ml})$. Such procedure was repeated for three times. Finally, the powders were washed with diethyl ether $(100 \mathrm{ml})$ to remove all residuals.

\section{Composites preparation}


The composites consisting of the neat or polymers modified GO particles and Vistamaxx matrix were mixed using Brabender (Duisburg, Germany). The device has three heating zones all operating at temperature $190{ }^{\circ} \mathrm{C}$, The components (filler, matrix) were added within one minute and whole compounding time was four minutes at speed $50 \mathrm{rpm}$.

\section{Characterization methods}

Molar mass $\left(M_{\mathrm{n}}\right)$ and dispersity $(\bigoplus)$ of synthesized polymer brushes were investigated by GPC, PL-GPC220 (Agilent, Japan) using polystyrene calibration and anisole as an internal standard. The resulting molar masses of poly(methyl methacrylate) (PMMA) and poly $(n-$ butyl acrylate) (PBA) were recalculated using Mark-Houwink parameters for PS ( $K=$ $\left.16.0 \cdot 10^{-5} \mathrm{dL} \mathrm{g}^{-1}, \alpha=0.700\right)$ [22], for PBA $\left(K=11.0 \cdot 10^{-5} \mathrm{dL} \mathrm{g}^{-1}, \alpha=0.723\right)$ and for PMMA $\left(K=12.8 \times 10^{-5} \mathrm{dL} \cdot \mathrm{g}^{-1}\right.$ and $\left.\alpha=0.690\right) .[23]$ Conversions of the monomers were calculated from ${ }^{1} \mathrm{H}$ NMR signals obtained from measurements VNMRS Varian spectrometer, $400 \mathrm{MHz}$ at $25^{\circ} \mathrm{C}$, and $\mathrm{CDCl}_{3}$ was used as a solvent. The chemical composition of neat and polymers modified GO was measured using Nicolet iS10 (Thermo Scientific, USA) connected to the TGA (TA, instruments), where the decomposed components were individually analyzed. The Raman shift was investigated from 3 scans (resolution of $2 \mathrm{~cm}^{-1}$ ) using a Nicolet DXR (Nicolet, USA). The individual layers of the GO and GO-polymer hybrids were analyzed using transmission electron microscopy (TEM) (Philips CM12, Nederlands). The XPS analysis chamber was evacuated approximately $6 \times 10-8 \mathrm{~Pa}$. The samples were excited by X-

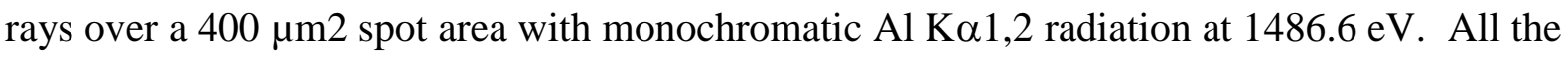
spectra were referenced to the main $\mathrm{C} 1 \mathrm{~s}$ peak of the carbon atoms, which was assigned a value of $284.8 \mathrm{eV}$. Samples for electrical conductivity in form of powder, were pressed on the hydraulic press (H-62, Trystom, Czech Republic). Then current responses have been evaluated using electrometer (Keithley 6517B, USA) and 10 measurement were used to 
provide final value. In order identify the density of the GO-based samples, the pellets were weight on air and in decane using Sartorius R 160P balances (Sartorius AG, Germany). The thermal conductivity was measured by one side contact method using TCi model (C-term technologies, Canada)

The rheological characteristics of composites were measured utilizing rheometer Physica (MCR502, Anton Paar, Austria) connected to Peltier heating/cooling fixture and parallelplate geometry (PP25). Samples in form of discs with thickness of $1 \mathrm{~mm}$ were elucidated. Firstly, the linear viscoelastic region was investigated. Then to avoid the sample slippage, the constant $0.3 \mathrm{~N}$ force was applied. Finally the frequency sweep in the range from $10^{-1}$ to $10^{1} \mathrm{~Hz}$ was performed. Final, values used in the figures are average values obtained from three individual measurements. In order to investigate the photo-responsive properties of neat polymer matrix and prepared nanocomposites in form of fibre mats the thermo-mechanical analyzer (TMA) (Mettler Toledo) [16]. The photo-actuation performance is ability of the material shows the reversible contraction and elongation upon irradiation with light source having $627 \mathrm{~nm}$ wavelength and intensity of $6 \mathrm{~mW}, 9 \mathrm{~mW}$ and $12 \mathrm{~mW}$. Temperature during the measurement was checked by external sensor and during whole investigation was $25^{\circ} \mathrm{C} \pm 0.4^{\circ} \mathrm{C}$

\section{Results and Discussion}

The coating of the GO polymer hybrids was successfully performed and reaction mixture based on sacrificial initiator was analyzed using GPC and ${ }^{1} \mathrm{H}$ NMR. The ratio of the individual components and characterization of the final polymers are presented in Table 1. Here, the polymers with narrow $Ð$ were obtained as a result of good control over the 
polymerization process. Finally, the GPC traces of both modifications can be seen in Fig. S1 (ESI).

Table 1: Composition of the polymerization mixture in molar ratios and characterization of the polymers.

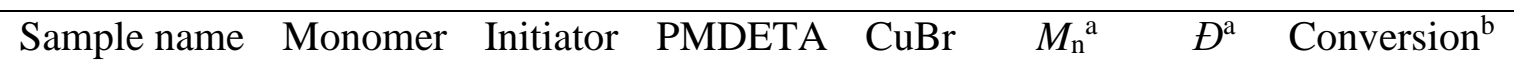

$\left(\mathrm{g} \mathrm{mol}^{-1}\right)$

\begin{tabular}{llllllll}
\hline GO-PMMA & 100 & 1 & 4 & 1 & 6800 & 1.18 & 89 \\
GO-PBMA & 100 & 1 & 4 & 1 & 5800 & 1.21 & 41
\end{tabular}

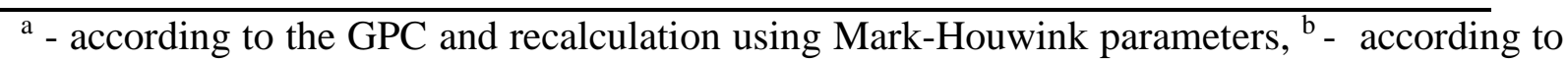
the ${ }^{1} \mathrm{HNMR}$

TEM images were investigated to get information about exfoliation of neat GO as well as GO-polymers hybrids. As can be in Fig. 1a the neat GO has several layers and exhibits expected 2D structure. SI-ATRP provides well-precise and controlled polymerization conditions with progressive growing the polymer chains from the GO surface and thus the 2D structure is retained for both GO-PMMA and GO-PBMA (Fig. 1b and 1c, respectively). The flossy-like appearance confirms the grafting of GO surface by very thin polymer layer. 


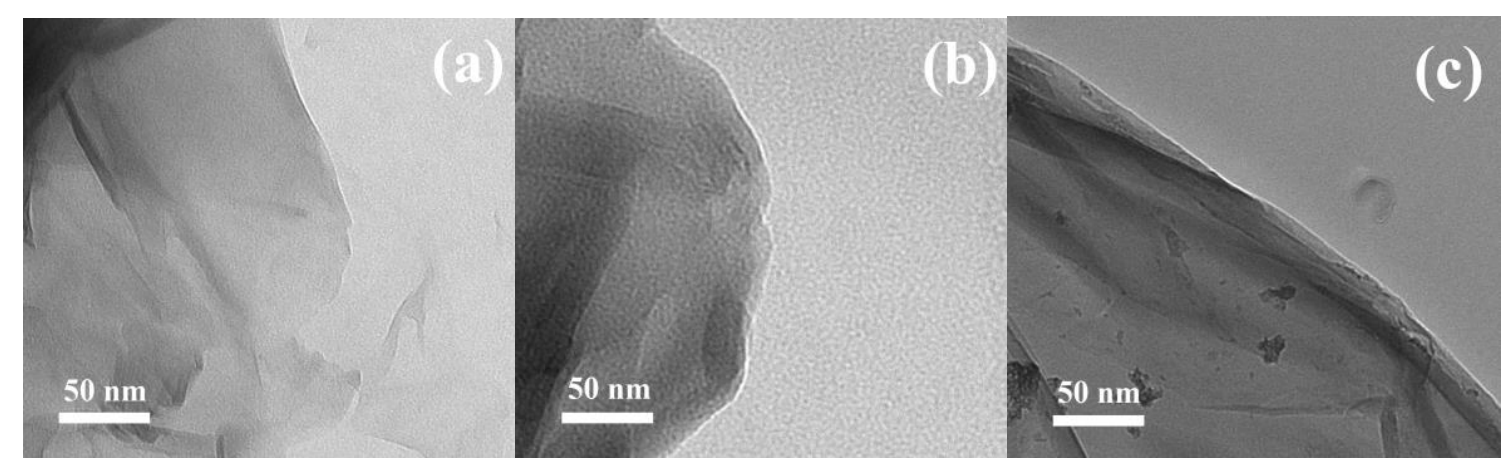

Fig. 1: TEM micrographs of the neat GO sheet(a), GO with PMMA brushes (b), and GO-PBMA brushes (c).

The investigation of the chemical composition of GO-based samples was performed using FTIR monitoring of the gas phase created during decomposition process of TGA. As can be seen in the Fig. $2 \mathrm{a}$ (neat GO), the main drop between $150-300{ }^{\circ} \mathrm{C}$ correspond to the stretching from hydroxyl and cabonyl moieties at $3510 \mathrm{~cm}^{-1}$ and $1723 \mathrm{~cm}^{-1}$. In addition in-plane bending vibration signal of $\mathrm{C}-\mathrm{OH}$ from carboxyl pendant was observed at $1423 \mathrm{~cm}^{-1}$. Controllable coating of GO sheets with short brushes shifted the release of oxygen moieties to lower temperatures. Furthermore, the decomposition of the polymers can be visible above $220^{\circ} \mathrm{C}$ (Fig. 2b and 2c). For GO-PMMA the FTIR collected at the temperature range of $220-$ $350{ }^{\circ} \mathrm{C}$ showed strong signals from stretching vibrations of carbonyl groups at about $1731 \mathrm{~cm}^{-1}$ and from C-H moieties at wavenumbers range of $1250-1550 \mathrm{~cm}^{-1}$ (Fig. 2e). In addition, new signals from stretching of alkyl moieties and bending of C-O-C moieties appeared at $2600-3000 \mathrm{~cm}^{-1}$ and also $1000-1200 \mathrm{~cm}^{-1}$, respectively, confirming presence of PMMA. Comparable signals were observed in FTIR spectra of degradation products collected at the temperature range of $220-380{ }^{\circ} \mathrm{C}$ from TGA of GO-PBMA (Fig. 2f), when PBMA moieties shows decomposition at higher temperatures. with the difference of shifting of the release of the groups from PBMA to the higher temperatures. Moreover, also the grafting density in both cases GO-PMMA as well as GO-PBMA were calculated according to 
the reference [15] and it was found that the grafting density was $0.83 \mathrm{chain} / \mathrm{nm}^{2}$ and 0.6 chain $/ \mathrm{nm}^{2}$, respectively.
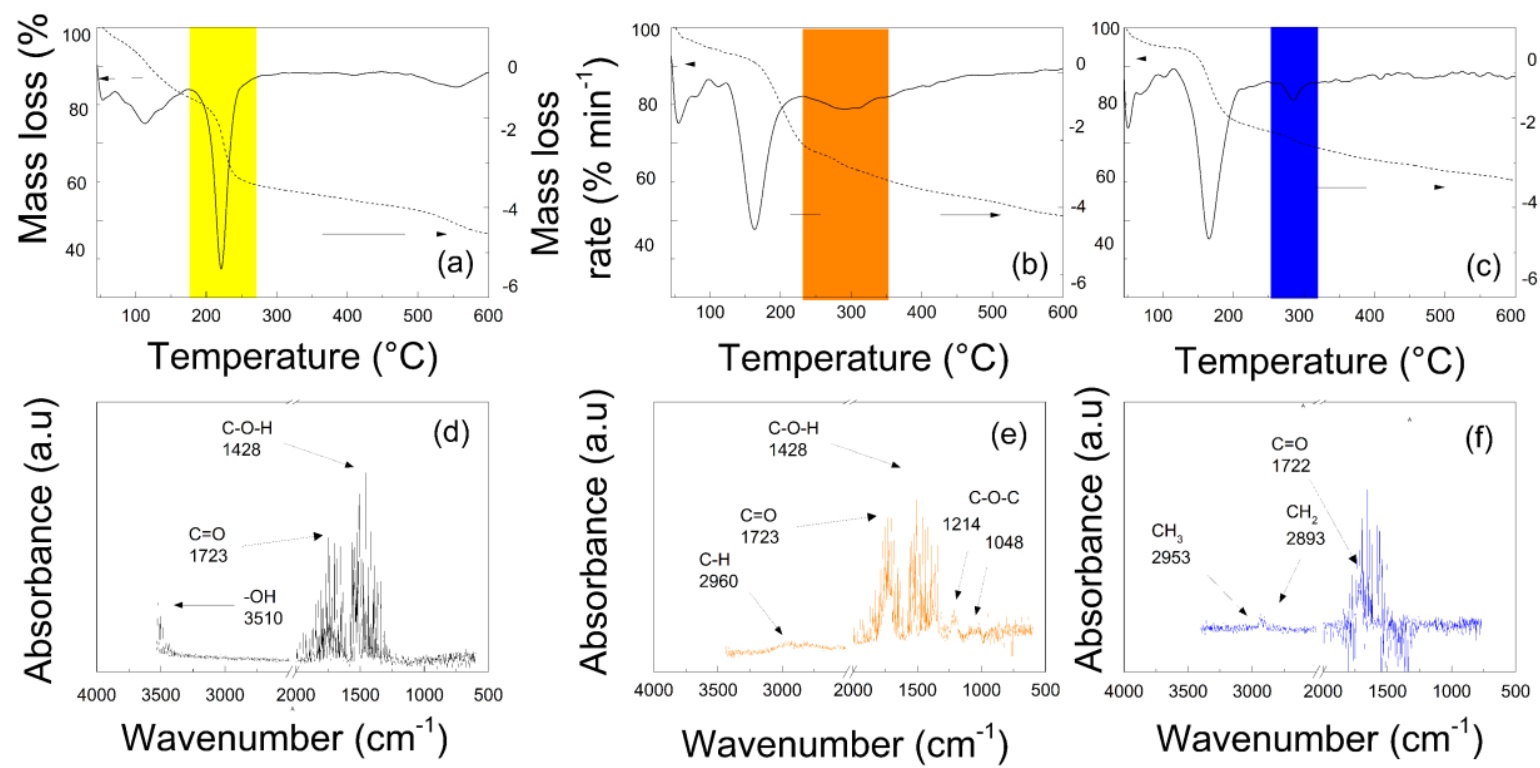

Fig. 2: TGA-FTIR online monitoring of the neat GO (a, d), GO-PMMA (b, e) and GO-PBMA (c, f) particles.

To further prove the covalent bonding of the polymer chains on GO surface the XPS spectra were measured and investigated (Fig. 3, Table 1). Following the successful coating with PMMA and PBMA chains, a clear increase in $\mathrm{C} / \mathrm{O}$ ratio could be observed due to higher carbon content in the polymers. This is more pronounced for PBMA where longer aliphatic chain is present. Moreover, increased content of $\mathrm{sp}^{2}$ accompanied by decreased content of $\mathrm{sp}^{3}$ hybridized form indicates that the reduction of GO surface took place as well, as will be further confirmed also by Raman shift and conductivity measurements. 

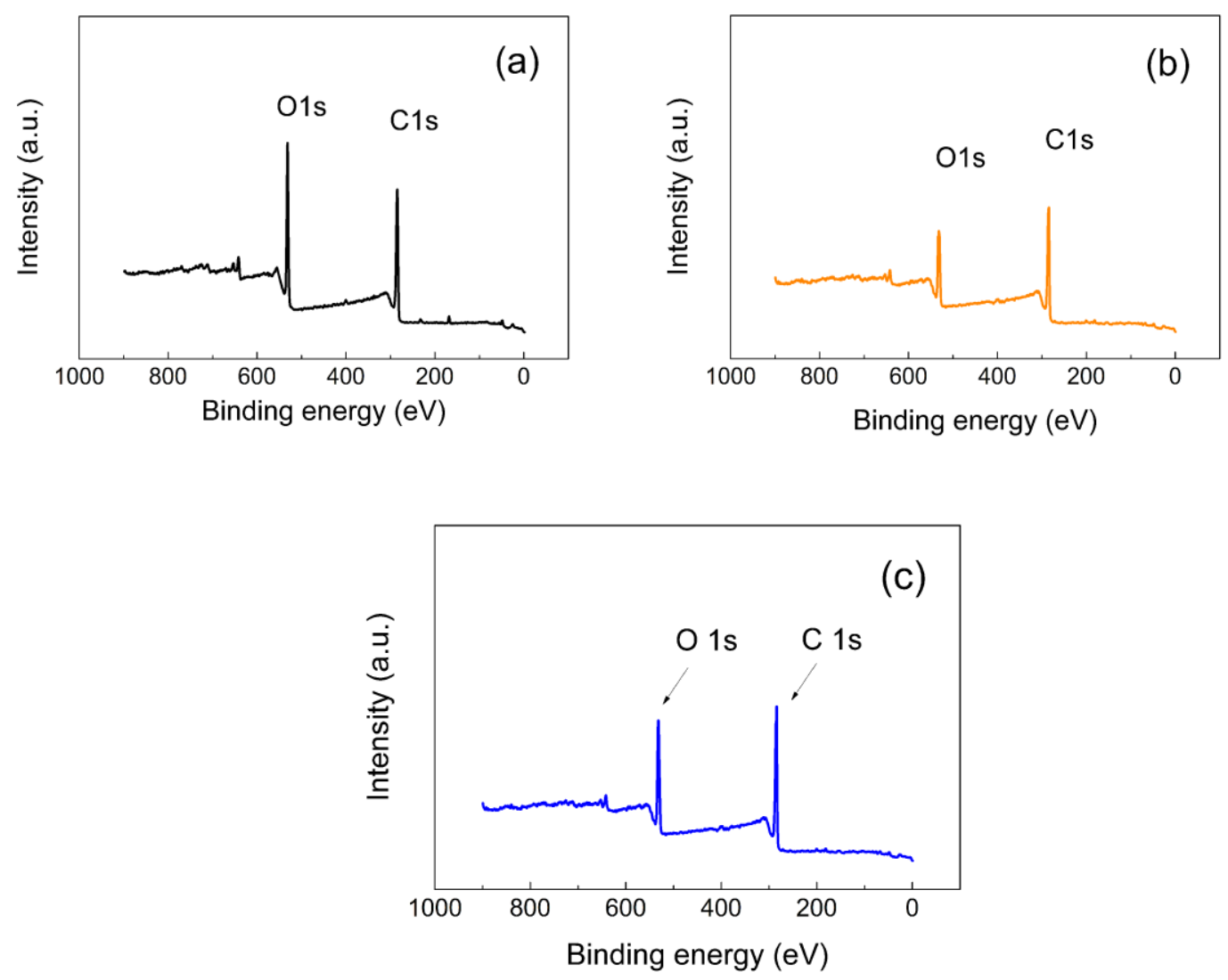

Fig. 3: XPS spectra of neat GO (a), GO-PMMA brushes (b) and GO-PBMA brushes (c).

Table 2: Surface element content (atomic \%) of neat GO and GO-polymers hybrids.

\begin{tabular}{l|l|l|l|l|l|l|l|l}
\hline Sample name & C1s & O1s & C1s sp & C1s sp & C1s C-O & C1s C=O & C1s O-C=O & C1s/O1s \\
\hline GO & 66.7 & 33.3 & 26.7 & 28.4 & 32.3 & 9.0 & 3.6 & 2.00 \\
GO-PMMA & 69.1 & 30.9 & 31.4 & 26.2 & 30.9 & 8.3 & 3.2 & 2.24 \\
GO-PBMA & 70.9 & 29.1 & 36.5 & 25.3 & 27.5 & 7.7 & 3.0 & 2.43 \\
\hline
\end{tabular}

\section{Reduction of the GO particles by SI-ATRP approach}

From the Raman spectra (Fig. 4a-c) is clearly shown that 2D structure of GO is negligibly affected by both polymer modifications (Fig. $4 \mathrm{e}$ and $4 \mathrm{f}$ ), while the considerable reduction of 
the GO surface was reached. This reduction was elucidate according to the calculations of the peak intensities those corresponding to $\mathrm{sp}^{2}$ and $\mathrm{sp}^{3}$ hybridization and are marked $\mathrm{I}_{\mathrm{D}}$ and $\mathrm{I}_{\mathrm{G}}$, respectively. This ratio is for neat GO 0.90 and for GO-PMMA 1.05 and GO-PBMA 1.08, showing usual increase when reduction of the GO surface takes place.

The comparative method for GO reduction evaluation is next to the abovementioned Raman, electric conductivity investigation those well-correlates with Raman reuslts. Electric conductivity increased from $1.2 \times 10^{-8} \mathrm{~S} \mathrm{~cm}^{-1}$ for neat GO, slightly increased to $6.3 \times 10^{-8} \mathrm{~S} \mathrm{~cm}^{-1}$ for GO-PMMA and $2.1 \times 10^{-7} \mathrm{~S} \mathrm{~cm}^{-1}$ for GO-PBMA. This is in good agreement with previously reported simultaneous partial GO surface reduction during controllable modification process. The controllable coating of graphene oxide surface by polymer chains was finally also confirmed by change of the GO density, which is decreased due to the certain polymer layer on the GO particles. Densities for investigated samples are summarized in the Table 3. 

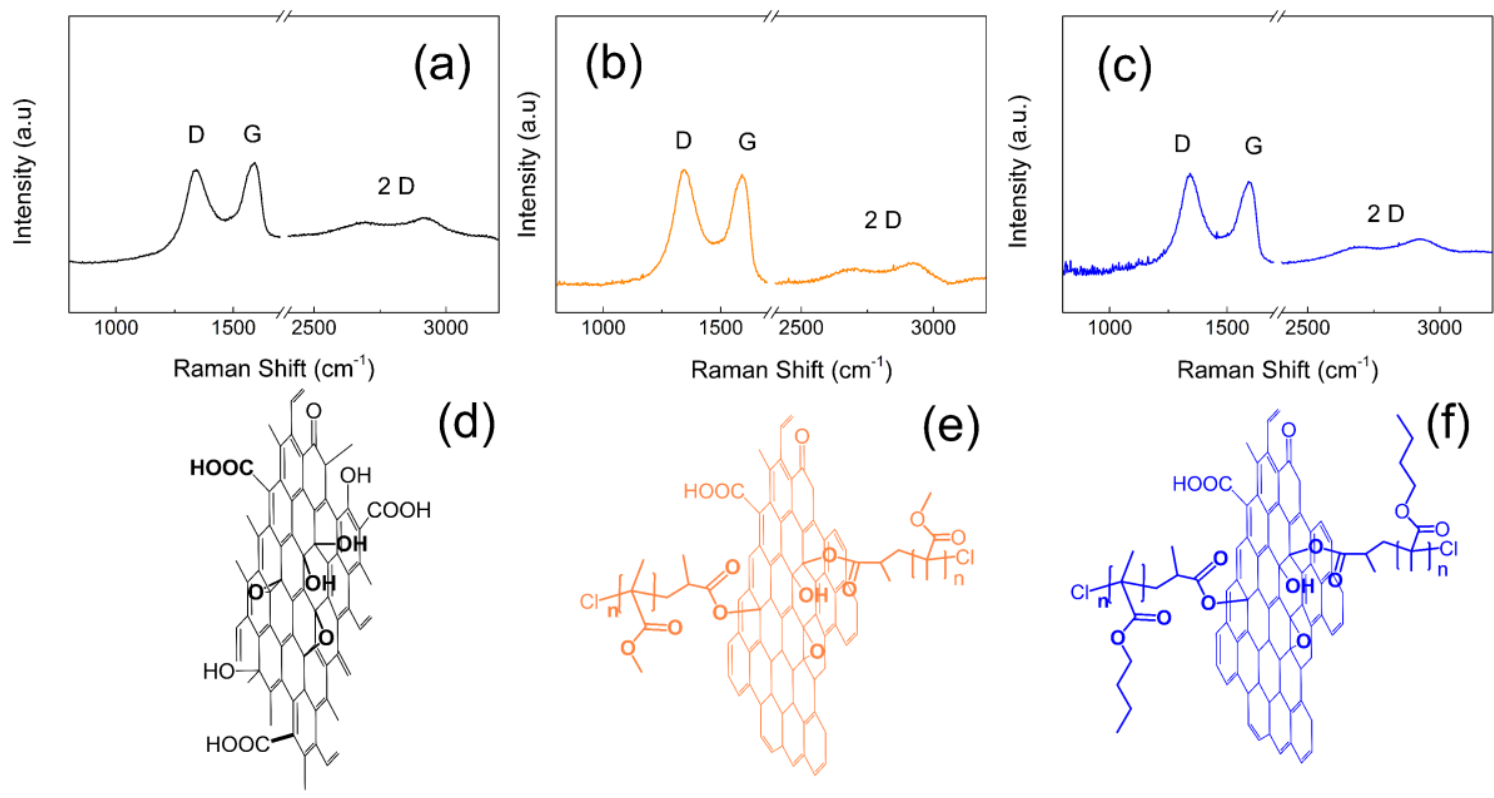

Fig. 4: Raman spectra of the neat GO (a), GO-PMMA (b) and GO-PBMA (c) and their schematic illustatrions for GO (d). GO-PMMA (e) and GP-PBMA (f).

Table 3: Conductivities and densities of neat GO and GO-modified particles.

\begin{tabular}{l|c|c}
\hline Sample name & Conductivity $\left(\mathrm{S} \mathrm{cm}^{-1}\right)$ & Density $\left(\mathrm{g} \mathrm{cm}^{-3}\right)$ \\
\hline neat GO & $1.210^{-8}$ & 2.68 \\
GO-PMMA & $6.310^{-8}$ & 2.53 \\
GO-PBMA & $2.110^{-7}$ & 2.34 \\
\hline
\end{tabular}

\section{Compatibility between the GO-polymer particles and Vistamaxx matrix}

The investigation of the viscoelastic properties at certain frequency range provide crucial information about compatibility of the modified GO sheets with the Vistamaxx copolymer It can be seen that neat Vistamaxx has cross-over point at $4.47 \mathrm{~Hz}$ (Fig. 5a), while for the composite containing neat GO particles cross-over point is $3.3 \mathrm{~Hz}$ (Fig. b) showing increases 
values of both moduli and reinforcing effect. For GO-PMMA and GO-PBMA grafted sheets cross-over points can be visible at $6.1 \mathrm{~Hz}$ and $6.30 \mathrm{~Hz}$, respectively, because of the short grafted brushes on the GO surface, providing the plasticizing effect for such composite in Vistamaxx matrix (Fig. 5c and 5d)). Similar results were observed for other thermoplastic elastomers $[24,25]$. The presence of the short polymer chains is highly beneficial in the case of our system, because it also significantly enhances flexibility of the composite [10]. Both these factors, i.e. better compatibility and higher flexibility, indicate that GO-polymer based composites should provide enhanced photo-actuation capability, the property which is investigated below.
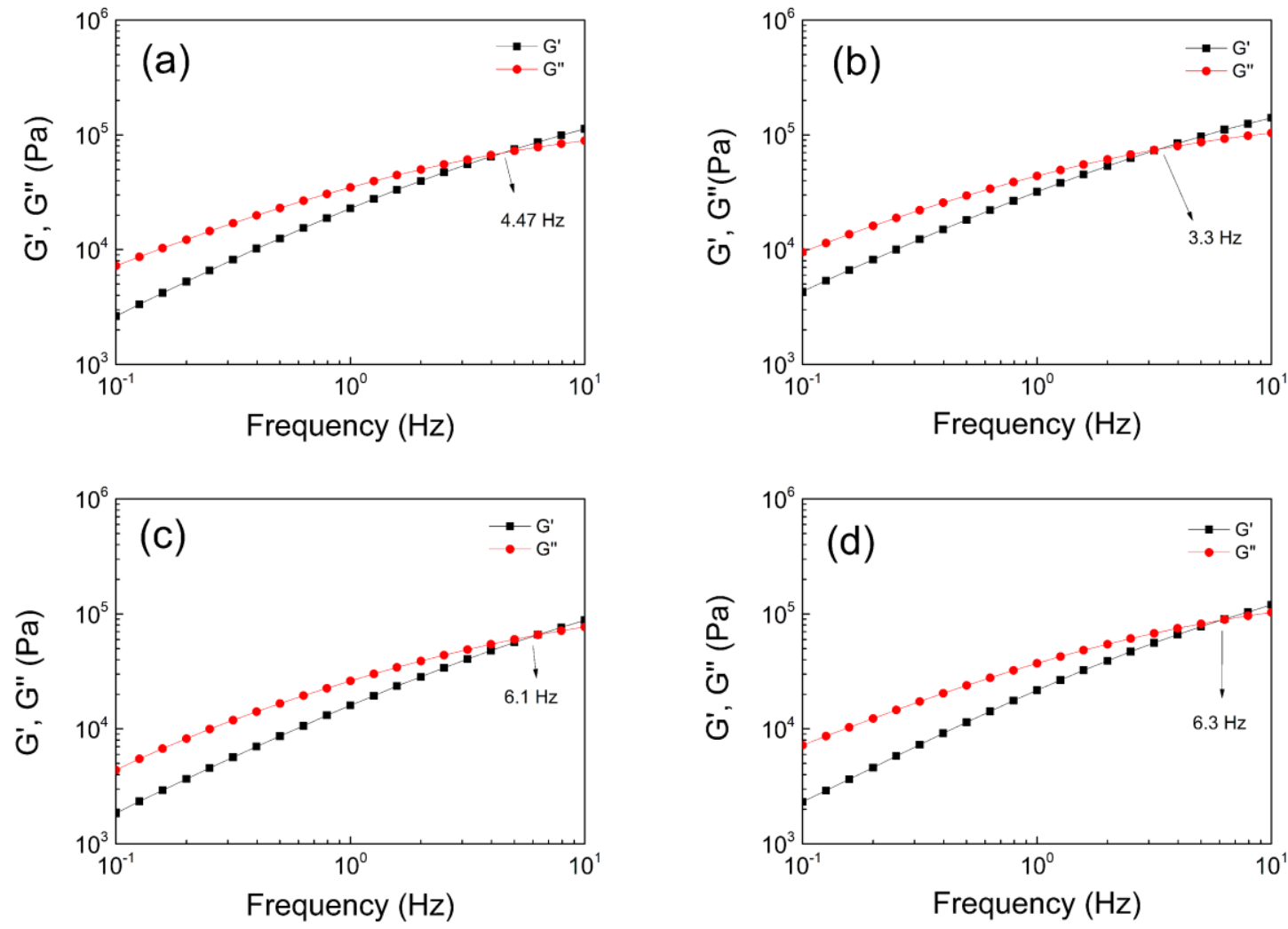

Fig. 5: Frequency dependence of storage, G', and loss G" moduli for neat Vistamaxx (a), and composites based on neat GO (b), GO-PMMA (c) and GO-PBMA (d) measured at $110^{\circ} \mathrm{C}$.

\section{Thermal Conductivity}


Thermal conductivity is very crucial factor for the material actuation upon light stimulation, due to the fact that, absorbed light from the source need to the redistributed within the whole sample and thus provide significantly improved actuation capability [19]. Table 4 shows the values of the thermal conductivity of various elastomeric composite samples. As was already confirmed by Raman spectroscopy and electric conductivity investigations, the electric conductivity of these system, increasing, which is also in correlation with thermal conductivity, which increased from 0.089 for neat matrix to 0.156 , 0.196 and $0.202 \mathrm{~W} \mathrm{mK}^{-1}$ for GO-filled, GO-PMMA and GO-PBMA filled elastomeric composites, respectively. Such increment is very promising from the photo-actuation point of view and will be further presented in the section below.

Table 4: Thermal conductivity of neat Vistamaxx and composites with GO-PMMA and GO-PBMA

\begin{tabular}{cc}
\hline Sample code & Thermal Conductivity $\left(\mathbf{W} \mathbf{~ m K}^{-\mathbf{1}}\right)$ \\
\hline Neat Vistamaxx & 0.089 \\
0.1 vol.\% GO & 0.156 \\
0.1 vol.\% GO-PMMA & 0.196 \\
0.1 vol.\% GO-PBMA & 0.202 \\
\hline
\end{tabular}

\section{Light-induced actuation capability}

Light -induced actuation capabilities of the prepared samples were investigated similarly as in our previous papers $[17,18]$, here also the effect of the GO coating on the actuation performance has been determined. Impact of the light intensity as well as filler loading in case of the most promising system was deeply elucidated. As is shown in Fig. 6, the neat 
Vistamaxx exhibit certain value of actuation, however the final actuation value, $\Delta L$, was $12.0 \mu \mathrm{m}$ with very long recovery time reaches nearly $30 \mathrm{~s}$. Photo-actuation performance was nearly doubled for composite with neat GO particles $(0.1$ vol. \%) due to ability of GO to absorb the light and the enhanced thermal conductivity of GO composite. After addition of 0.1 vol. $\%$ of GO-PMMA sheets, the absolute value of actuation reaches $30 \mu \mathrm{m}$ while time to reach the initial length decreased to $25 \mathrm{~s}$. More pronounced improvement can be seen for composite sample with GO-PBMA sheets, where absolute maximum value reaches $68 \mu \mathrm{m}$, and showing very similar recovery time as previous modification $(25 \mathrm{~s})$. The crucial factor which can be assign for significantly improved actuation performance is improved compatibility of polymer hybrids of GO and short PMMA and PBMA polymer brushes with Vistamaxx and also partial plasticizing effect on the matrix and improved heat transition within the samples causing easier and more proper shape recovery.

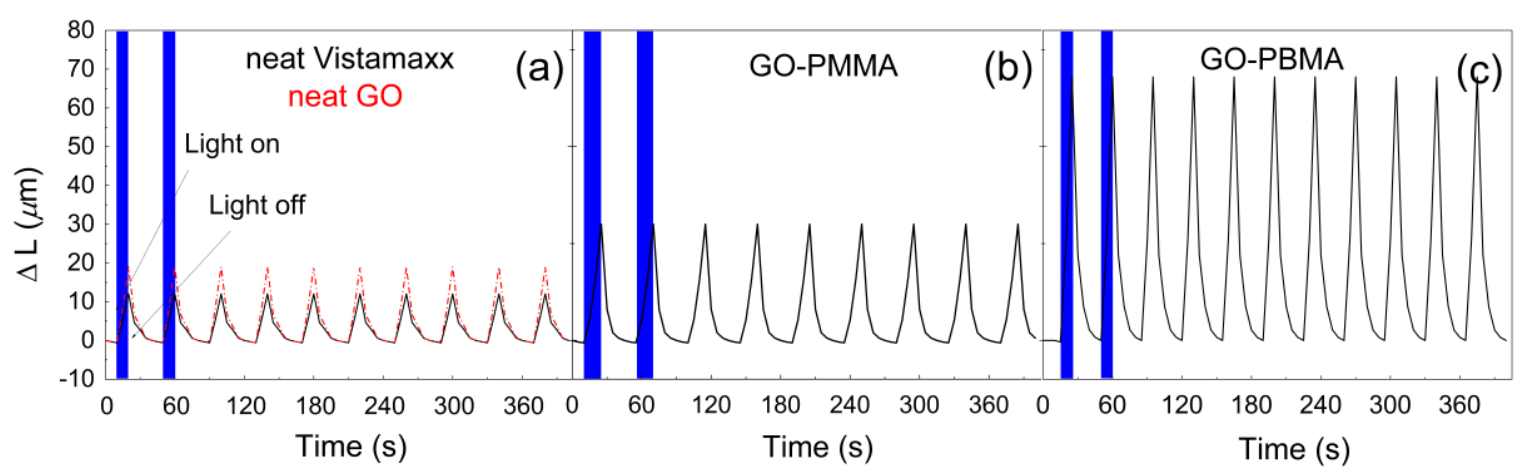

Fig. 6: Photo-actuation performance of neat Vistamaxx (black solid line) and composite contaning 0.1 vol. $\%$ of neat GO (red dash-dot line) (a), GO-PMMA (b), and GO-PBMA (c) at light intensity of $6 \mathrm{~mW}$.

Further the impact of light source intensity on the photo-actuation capability in case of Vistamaxx-based composites was elucidated and can be clearly seen in Fig. 7. Generally, the change in the length increasing when the light source intensity increases. From this point of view the composites with GO-PBMA sheets, were investigated, due to the fact that exhibit 
the best actuation performance at $6 \mathrm{~mW}$. However if the light intensity was increased to $12 \mathrm{~mW}$, the actuation performance reaches $110 \mu \mathrm{m}$, what is about 8 times higher value than for neat Vistamaxx. The neat Vistamaxx as well as composite containing neat GO and GOPMMA shows certain saturation of the photo-actuation properties, since the increment of the change in the length is slightly decreasing with increasing light source power. This is not a case of composite containing GO-PBMA particle; this composite has similar change in the length increment with increasing light source power and at highest power provide unsaturated system. At this point can be stated that significantly increased flexibility of the composite system can be assigned to the presence of short polymer brushes, which finally contribute to the considerable enhancement of actuation performance [24-26]. The GO modification also significantly improved the compatibility with Vistamaxx matrix providing samples with proper filler distribution improving the final thermal conductivity and thus the transformation of the energy from light to mechanical movement. GO-based systems showed in this study have comparably higher actuation performance in comparison to CNTs, carbon black, GO or graphene nanoplatelets (2 wt.\%) in the PDMS composites. Those samples exhibit only $25 \mu \mathrm{m}$ actuation recalculated to our conditions [27]. 


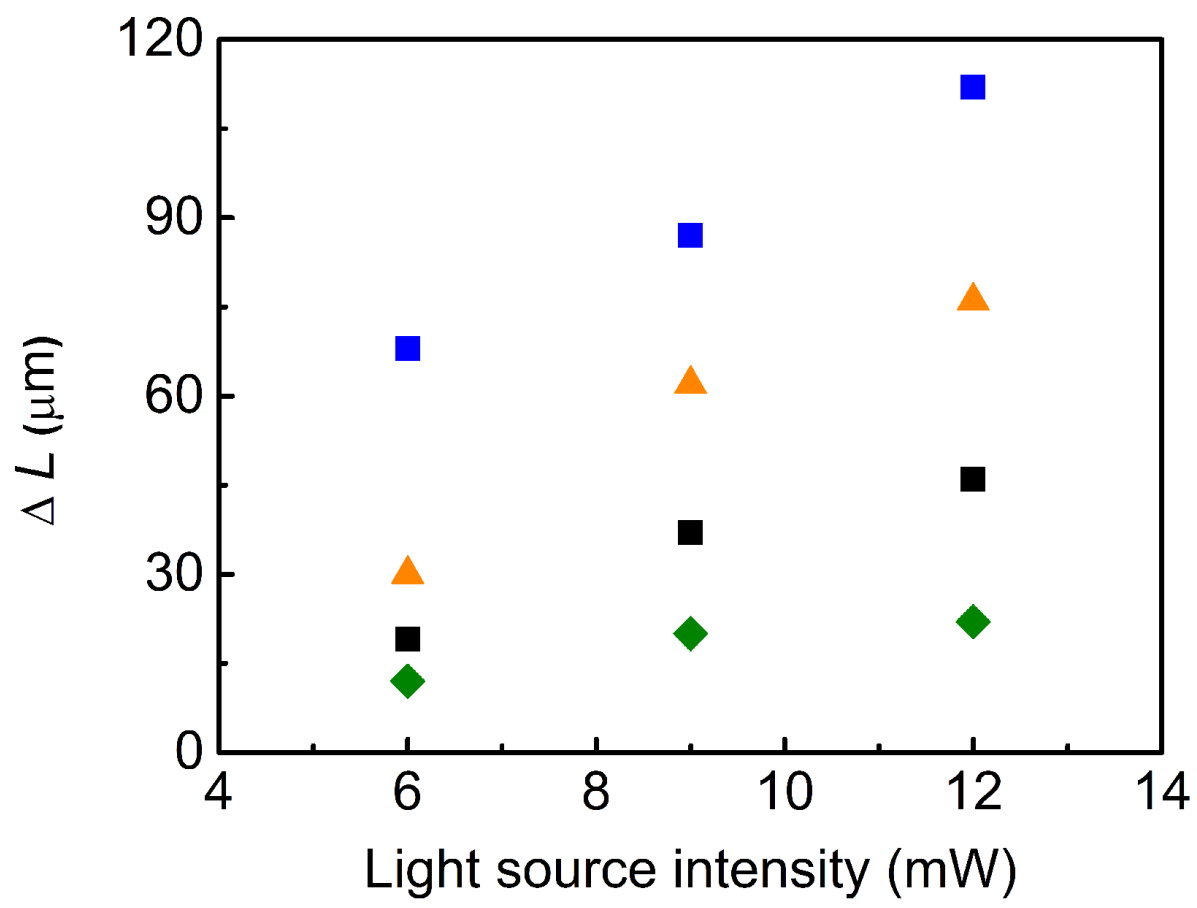

Fig. 7: Dependence of the change in the length on the light source power for neat Vistamaxx matrix (green diamonds) and composites containing 0.1 vol. \% of neat GO (black squares), GO-PMMA (orange triangles) and GO-PBMA (blue squares). Height of the symbols are corresponding to the error bars of the measurement

Increasing the filler content from 0.1 vol. $\%$ to 0.5 and 1 vol. $\%$ led to increased photoactuation capabilities, while the course was different for individual systems based on the type of dispersed particles (Fig. 8). Change in the length for GO-based composites reached $29 \mu \mathrm{m}$ at 0.5 vol. $\%$ and just slightly increased for 1 vol. $\%$ to $37 \mu \mathrm{m}$. Similarly, for the composite with GO-PMMA particles the increment in the change in the length with particles concentration was not linear but slightly saturates and reached values of $56 \mu \mathrm{m}$ and $75 \mu \mathrm{m}$ for 0.5 and 1 vol. \%, respectively. The highest photo-actuation performance was again discovered for samples including GO-PBMA polymer hydrides. As can be seen the change in the length increment is still linear and suggests that further filler loading could be effective and actuation performance could be higher than obtained for 0.5 and 1 vol. $\%, 91 \mu \mathrm{m}$ and $118 \mu \mathrm{m}$, which is high enough for application as a part of tactile displays. These results confirm the previous findings, that flexibility of the matrix together with proper filler 
dispersion and enhanced thermal conductivity are crucial factor influencing the photoactuation capability.

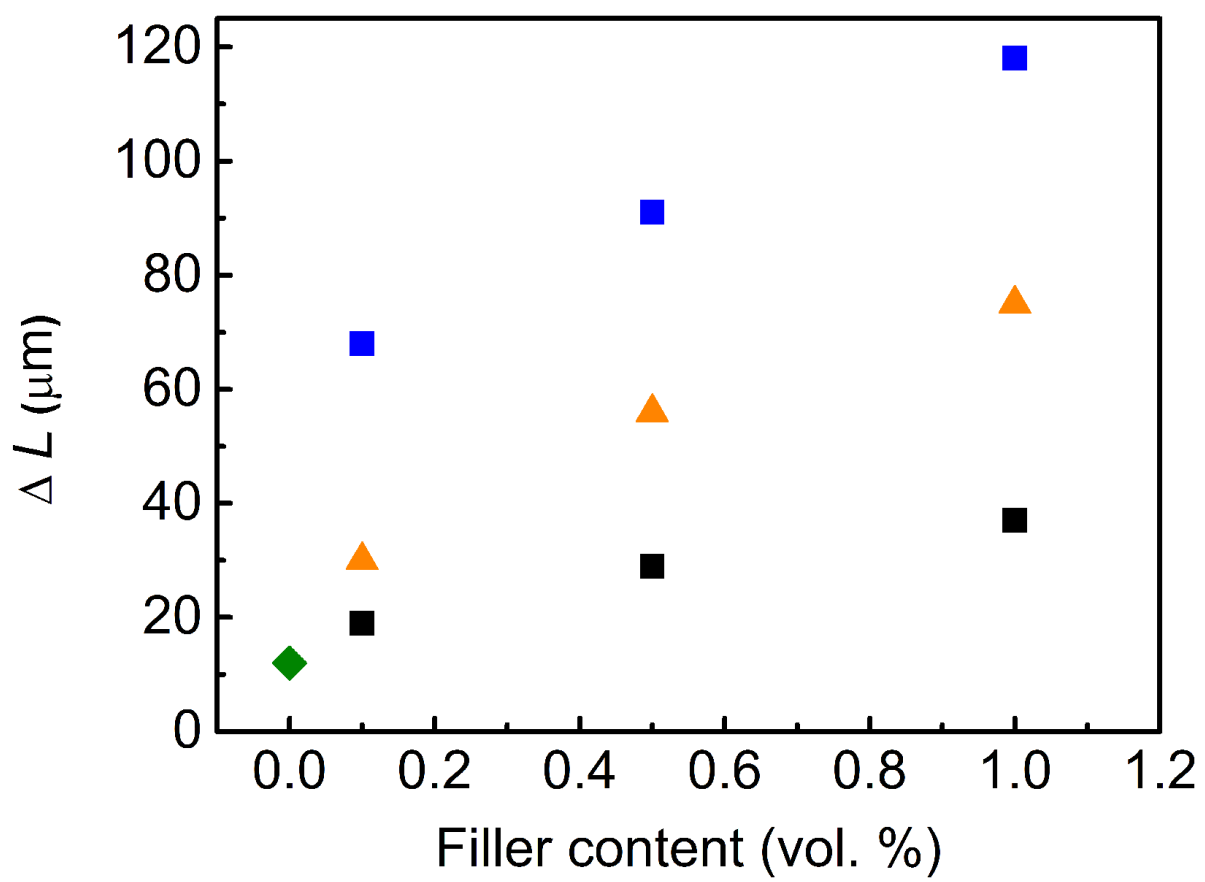

Fig. 8: Dependence of the change in the length on the filler content for neat Vistamaxx matrix (green diamonds) and vistamaxx elastomers with GO particles (black squares), GO-PMMA (orange triangles) and GO-PBMA (blue squares) at light intensity of $6 \mathrm{~mW}$. Height of the symbols are corresponding to the error bars of the measurement.

\section{Conclusions}

This study is dealing with the influence of the modification of the GO particles on the photoactuation performance of the thermoplastic elastomer matrix. The SI-ATRP approach was utilized for grafting of PMMA and PBMA chains from the GO sheets accompanied with controllable reduction. The grafting was proved using TEM, GPC, ${ }^{1} \mathrm{H}$ NMR, TGA-FTIR and XPS. The partial GO reduction was confirmed with Raman shift, electric conductivity investigations and XPS. The improved interactions between of the GO- based polymer brushes with Vistamaxx was proved by melt rheology investigation where the crossover point 
was shifted to the higher frequencies and showed enhanced compatibility. Finally the photoactuation studies showed the excellent length change values of $120 \mu \mathrm{m}$ for $6 \mathrm{~mW}$ at $1 \mathrm{vol} . \%$ of GO-PBMA loading and nearly $110 \mu \mathrm{m}$ at $12 \mathrm{~mW}$ for only 0.1 vol.\% of GO-PBMA loading. This photo-actuation capability is significantly higher than obtained previously for other similar systems. Thus it has to be concluded that the approach reported here is very promising for further investigation and possible increased filler loading can further enhance the photo-actuation capability.

\section{Acknowledgement}

This research was funded by Qatar University Collaborative High Impact Grant QUHICENG-18/19-1. The findings achieved herein are solely the responsibility of the authors. Authors also gratefully thanks to the Grant Agency of the Czech Republic (no. 16-20361Y) for financial support. This work was also supported by the Ministry of Education, Youth and Sports of the Czech Republic - program NPU I (LO1504). This study was also performed during the implementation of the project Building-up Centre for advanced materials application of the Slovak Academy of Sciences, ITMS project code 313021T081 supported by Research \& Innovation Operational Programme funded by the ERDF. JM also acknowledge for financial support to project VEGA 2/0129/19. This article (specify by the fact) was also written with support of Operational Program Research and Development for innovations co-funded by the European Regional Development Fund (ERDF) and national budget of Czech Republic, within the framework of project CPS - strengthening research capacity (reg. number: CZ.1.05/2.1.00/19.0409). M. I. also thank to National Science Centre, Poland for the financial support through POLONEZ project number UMO2016/23/P/ST5/02131. This project is carried out under POLONEZ program which has 
received funding from the European Unions's Horizon 2020 research and innovation programme under Marie Sklodowska-Curie grant agreement. No 665778.

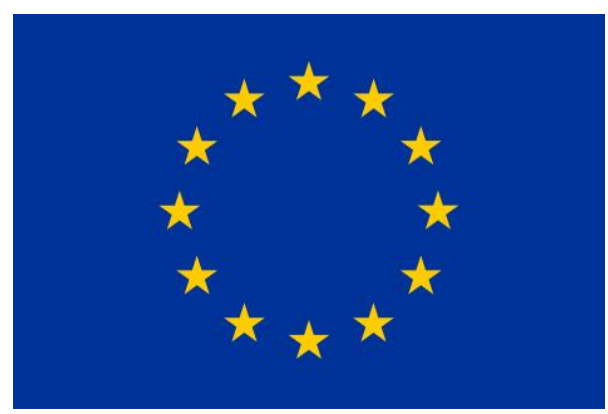

\section{References}

1. Y. Hu, G. Wu, T. Lan, J. J. Zhao, Y. Liu, W. Chen, A Graphene-Based Bimorph Structure for Design of High Performance Photoactuators, Advanced Materials, 2015, 27, 786710.1002/adma.201502777

2. Y. Hu, Z. Li, T. Lan, W. Chen, Photoactuators for Direct Optical-to-Mechanical Energy Conversion: From Nanocomponent Assembly to Macroscopic Deformation, Adv. Mater., 2016, 28, 1054810.1002/adma.201602685

3. X. Zhang, Z. B. Yu, C. Wang, D. Zarrouk, J. W. T. Seo, J. C. Cheng, A. D. Buchan, K. Takei, Y. Zhao, J. W. Ager, J. J. Zhang, M. Hettick, M. C. Hersam, A. P. Pisano, R. S. Fearing, A. Javey, Photoactuators and motors based on carbon nanotubes with selective chirality distributions, Nat. Commun., 2014, 5, 82983,10.1038/ncomms3983

4. Leeladhar, P. Raturi, J. P. Singh, Sunlight-driven eco-friendly smart curtain based on infrared responsive graphene oxide-polymer photoactuators, Sci Rep, 2018, 8, 93687,10.1038/s41598-01821871-3

5. Y. Hu, J. Q. Liu, L. F. Chang, L. L. Yang, A. F. Xu, K. Qi, P. Lu, G. Wu, W. Chen, Y. C. Wu, Electrically and Sunlight-Driven Actuator with Versatile Biomimetic Motions Based on Rolled Carbon Nanotube Bilayer Composite, Adv. Funct. Mater., 2017, 27, 101704388,10.1002/adfm.201704388

6. D. Kim, H. S. Lee, J. Yoon, Highly bendable bilayer-type photo-actuators comprising of reduced graphene oxide dispersed in hydrogels, Sci Rep, 2016, 6, 1020921,10.1038/srep20921

7. L. L. Yang, K. Qi, L. F. Chang, A. F. Xu, Y. Hu, H. Zhai, P. Lu, A powerful dual-responsive soft actuator and photo-to-electric generator based on graphene micro-gasbags for bioinspired applications, J. Mat. Chem. B, 2018, 6, 910.1039/c8tb01222a 
8. Leeladhar, P. Raturi, A. Kumar, J. P. Singh, Graphene-polydimethylsiloxane/chromium bilayer-based flexible, reversible, and large bendable photomechanical actuators, Smart Mater. Struct., 2017, 26, 9095030,10.1088/1361-665X/aa7a49

9. L. Yu, and H. F. Yu, Light-Powered Tumbler Movement of Graphene Oxide/Polymer Nanocomposites, ACS Appl. Mater. Interfaces, 2015, 7, 383410.1021/am508970k

10. M. Ilcikova, M. Mrlik, T. Sedlacek, D. Chorvat, I. Krupa, M. Slouf, K. Koynov, J. Mosnacek, Viscoelastic and photo-actuation studies of composites based on polystyrene-grafted carbon nanotubes and styrene-b-isoprene-b-styrene block copolymer, Polymer, 2014, 55, 21110.1016/j.polymer.2013.11.031

11. R. Rohini, P. Katti, S. Bose, Tailoring the interface in graphene/thermoset polymer composites: A critical review, Polymer, 2015, 70, A1710.1016/j.polymer.2015.06.016

12. M. Mrlik, M. Ilcikova, T. Plachy, R. Moucka, V. Pavlinek, J. Mosnacek, Tunable electrorheological performance of silicone oil suspensions based on controllably reduced graphene oxide by surface initiated atom transfer radical polymerization of poly(glycidyl methacrylate), Journal of Industrial and Engineering Chemistry, 2018, 57, 104

13. M. Mrlik, M. Ilcikova, T. Plachy, V. Pavlinek, Z. Spitalsky, J. Mosnacek, Graphene oxide reduction during surface-initiated atom transfer radical polymerization of glycidyl methacrylate: Controlling electro-responsive properties, Chem. Eng. J., 2016, 283, 71710.1016/j.cej.2015.08.013

14. M. Ilcikova, M. Mrlik, Z. Spitalsky, M. Micusik, K. Csomorova, V. Sasinkova, A. Kleinova, J. Mosnacek, A tertiary amine in two competitive processes: reduction of graphene oxide vs. catalysis of atom transfer radical polymerization, RSC Adv., 2015, 5, 337010.1039/c4ra12915f

15. C. M. Hui, J. Pietrasik, M. Schmitt, C. Mahoney, J. Choi, M. R. Bockstaller, K. Matyjaszewski, Surface-Initiated Polymerization as an Enabling Tool for Multifunctional (Nano-)Engineered Hybrid Materials, Chemistry of Materials, 2014, 26, 74510.1021/cm4023634

16. S. T. Milner, POLYMER BRUSHES, Science, 1991, 251, 90510.1126/science.251.4996.905

17. J. Osicka, M. Mrlik, M. Ilcikova, B. Hanulikova, P. Urbanek, M. Sedlacik, J. Mosnacek, Reversible Actuation Ability upon Light Stimulation of the Smart Systems with Controllably Grafted Graphene Oxide with Poly (Glycidyl Methacrylate) and PDMS Elastomer: Effect of Compatibility and Graphene Oxide Reduction on the Photo-Actuation Performance, Polymers, 2018, 10, $14832,10.3390 /$ polym 10080832

18. J. Osicka, M. Ilcikova, M. Mrlik, A. Minarik, V. Pavlinek, J. Mosnacek, The Impact of Polymer Grafting from a Graphene Oxide Surface on Its Compatibility with a PDMS Matrix and the LightInduced Actuation of the Composites, Polymers, 2017, 9, 14264,10.3390/polym9070264 
19. Y. Y. Feng, M. M. Qin, H. Q. Guo, K. Yoshino, W. Feng, Infrared-Actuated Recovery of Polyurethane Filled by Reduced Graphene Oxide/Carbon Nanotube Hybrids with High Energy Density, Acs Applied Materials \& Interfaces, 2013, 5, 1088210.1021/am403071k

20. J. Osicka, M. Mrlik, M. Ilcikova, B. Hanulikova, P. Urbanek, M. Sedlacik, J. Mosnacek, Reversible actuation ability upon light stimulation of the smart systems with controllably grafted graphene oxide with poly (glycidyl methacrylate) and PDMS elastomer: Effect of compatibility and graphene oxide reduction on the photo-actuation performance, Polymers, 2018, 10, $832,10.3390 /$ polym 10080832

21. W. S. Hummers, and R. E. Offeman, PREPARATION OF GRAPHITIC OXIDE, J. Am. Chem. Soc., 1958, 80, 133910.1021/ja01539a017

22. http://www.ampolymer.com/Mark-Houwink.html

23. T. Gruemdling, T. Junkers, M. Guilhaus, C. Barner-Kowollik, Mark-Houwink Parameters fo the Universal Calibration of the Acrylate and Vinyl Acetate Polymers Determined by Online SizeExclusion Chromatrography-Mass Spectroscopy, Macromol. Chem. Phys. 2010, 211, 10.1002/macp.200900323.

24. M. Ilcikova, M. Mrlik, T. Sedlacek, M. Slouf, A. Zhigunov, K. Koynov, J. Mosnacek, Synthesis of Photoactuating Acrylic Thermoplastic Elastomers Containing Diblock Copolymer-Grafted Carbon Nanotubes, ACS Macro Lett., 2014, 3, 99910.1021/mz500444m

25. M. Ilcikova, M. Mrlik, T. Sedlacek, M. Doroshenko, K. Koynov, M. Danko, J. Mosnacek, Tailoring of viscoelastic properties and light-induced actuation performance of triblock copolymer composites through surface modification of carbon nanotubes, Polymer, 2015, 72, 36810.1016/j.polymer.2015.03.060

26. M. Cvek, M. Mrlik, M. Ilcikova, J. Mosnacek, L. Munster, V. Pavlinek, Synthesis of Silicone Elastomers Containing Silyl-Based Polymer Grafted Carbonyl Iron Particles: An Efficient Way To Improve Magnetorheological, Damping, and Sensing Performances, Macromolecules, 2017, 50, 218910.1021/acs.macromol.6b02041

27. J. Loomis, B. King, T. Burkhead, P. Xu, N. Bessler, E. Terentjev, B. Panchapakesan, Graphenenanoplatelet-based photomechanical actuators, Nanotechnology, 2012, 23, 045501,10.1088/0957$4484 / 23 / 4 / 045501$ 